\title{
Silver telluride nanotubes prepared by hydrothermal
}

\section{method}

\author{
Aimiao Qin ${ }^{\dagger}$, Yueping Fang, ${ }^{*},+$ Pingfang Tao ${ }^{\dagger}$, Jianyong Zhang ${ }^{+}$, Chengyong Su, ${ }^{*}+\dot{t}$ \\ $\dagger$ School of Chemistry and Chemical Engineering, Guangxi Normal University, Guilin,Guangxi, \\ 541004, China. E-mail: fyp66115@tom.com; Fax: (86) 773-5803930; Tel: (86) 773-5821167 \\ $\$$ Key Laboratory of Bioinorganic and Synthetic Chemistry of MOE and State Key Laboratory of \\ Optoelectronic Materials and Technologies, School of Chemistry and Chemical Engineering, Sun Yat- \\ Sen University, Guangzhou 510275, China. Email: cesscy@mail.sysu.edu.cn
}

\section{Supporting Information}

Figure S1. A) TEM image of the as-prepared $\mathrm{Ag}_{2} \mathrm{Te}$ sample dispersed on copper grid after the exposure to laser beam for 30 minutes during the Raman spectra measurement; B) and C) magnified images; D) HRTEM image of a particle on the surface of sample and inset is live FFT pattern; E) HRTEM image of another particle and inset is magnified image taken from white square in (E); F) EDS spectrum recorded from white circle in (D), showing only Ag element; G) EDS spectrum recorded from as-prepared $\mathrm{Ag}_{2} \mathrm{Te}$ sample without the exposure to laser beam, showing Ag and Te elements; H) EDS spectrum recorded from white circle in (E), and showing Ag, Te and $\mathrm{O}$ elements. 


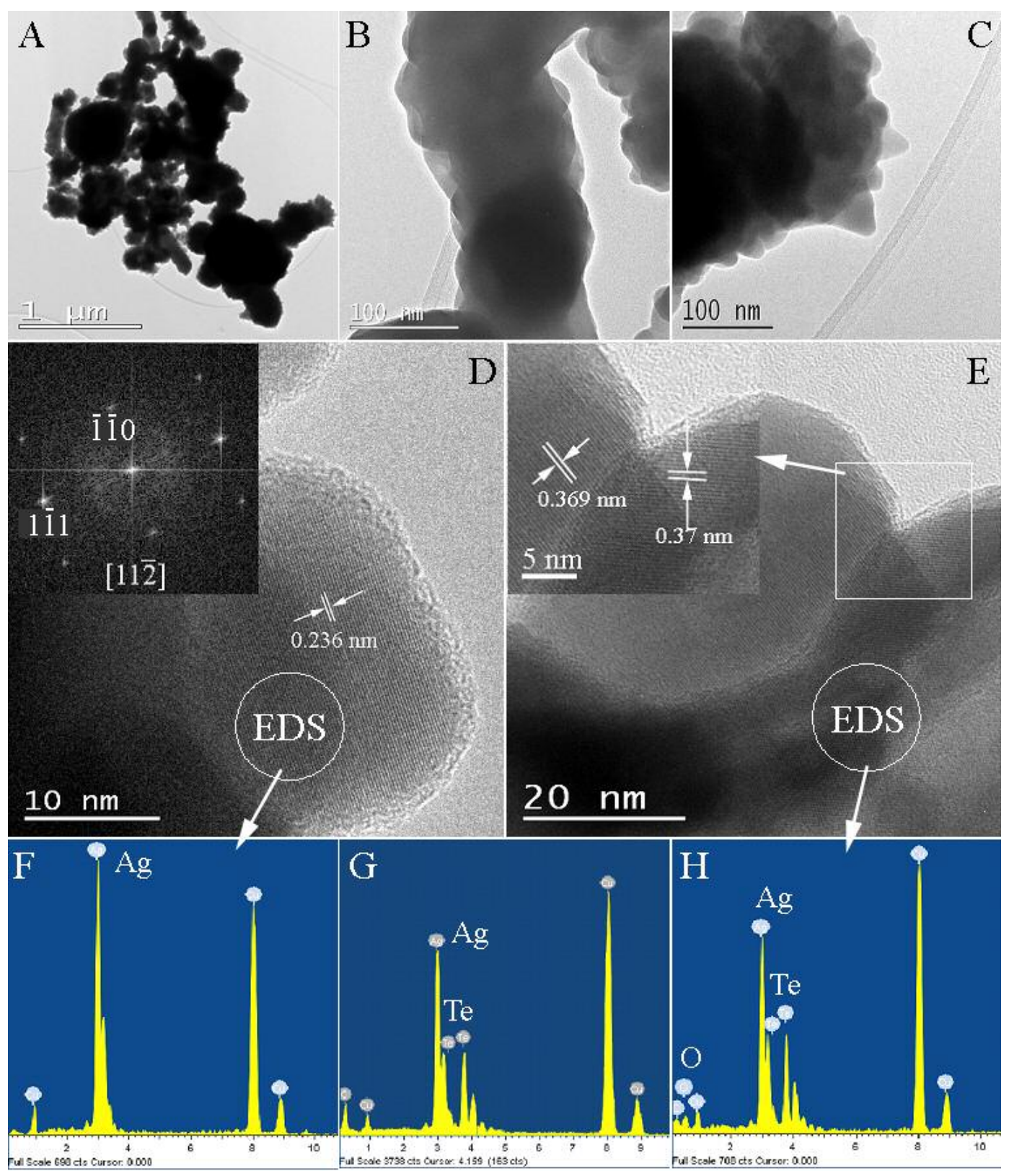


Figure S2. SEM images of the samples obtained by hydrothermal reaction after different reaction time.

A) 3-4 hours, white arrows denote nanobelts and curling-up nanobelts; B) 6-7 hours, inset is typical TEM image of individual half-tube; C) more than 9 hours; D) a SEM image of the obtained sample when the hydrothermal reaction was carried out in absence of $\mathrm{NH}_{3} \cdot \mathrm{H}_{2} \mathrm{O}$.

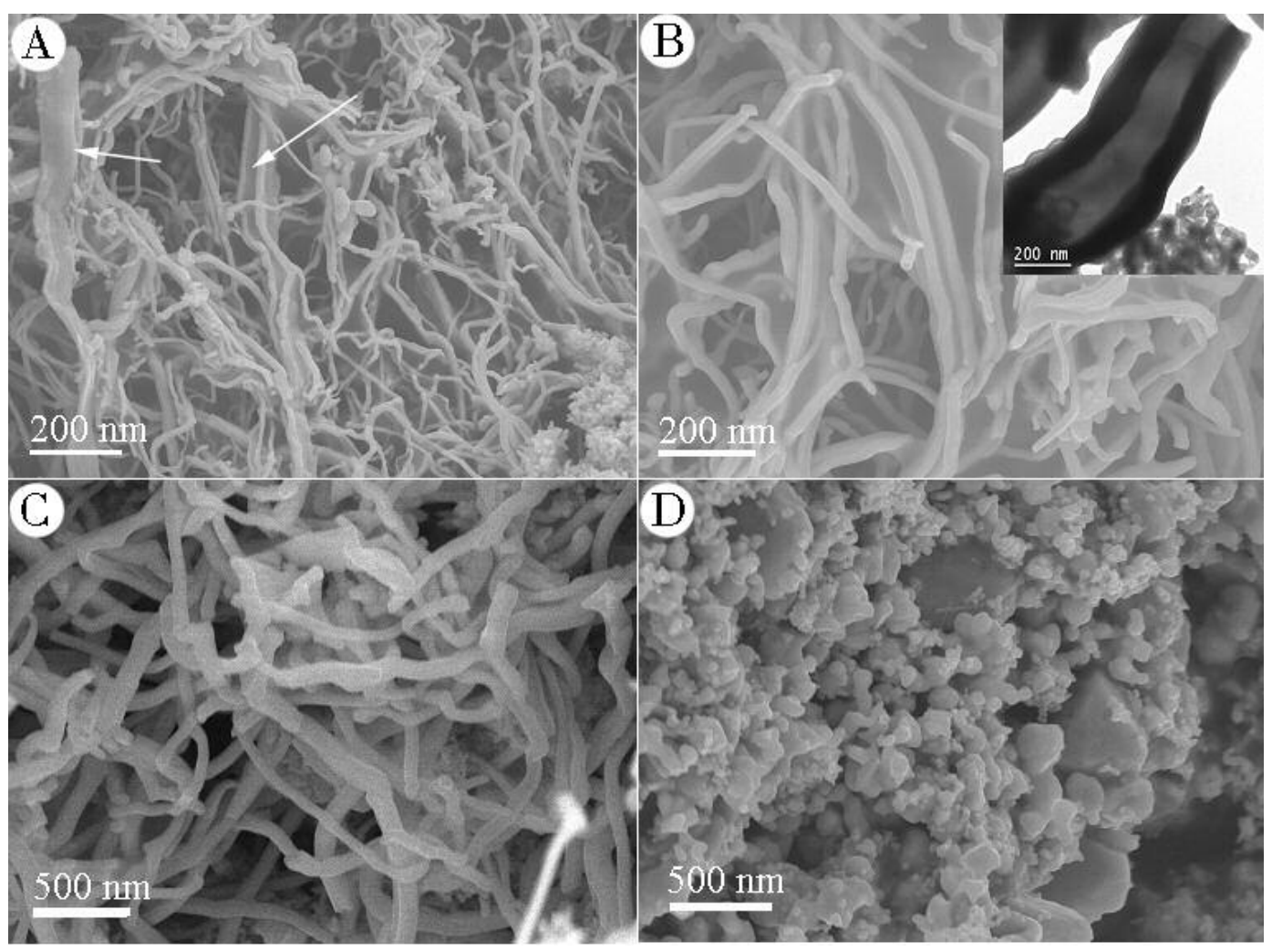

